\title{
TROCAS GASOSAS INTRAPULMONARES SOB RESPIRAÇÃO EM AR AMBIENTE EM PACIENTES HIPERCAPNEICOS
}

\author{
Alfredo Ribeiro-Silva, Geruza Alves da Silva* \\ Divisão de Pneumologia do Hospital das Clínicas da Faculdade de Medicina de Ribeirão Preto da \\ Universidade de São Paulo (HCFMRP/USP), Ribeirão Preto, SP.
}

RESUMO - A diferença alvéolo-arterial de oxigênio ou $\mathrm{P}(\mathrm{A}-\mathrm{a}) \mathrm{O}_{2}$ importante no estudo de disfunções nas trocas gasosas alvéolo-capilares, entretanto não fornece resultados confiáveis em pacientes hipercapneicos, ao contrário do cálculo da mistura venosa que, embora pouco prático, é potencialmente capaz de diagnosticar a existência de distúrbio de trocas alvélo-capilares de qualquer natureza.

Objetivo. Conferir o grau de confiabilidade da diferença alvéoloarterial de oxigênio para avaliar distúrbio das trocas alvéolo-capilares na presença de hipoventilação alveolar, utilizando o cálculo da mistura venosa como padrão.

Métodos. Oitenta e três gasometrias de pacientes hipercapneicos foram submetidas ao cálculo da mistura venosa e da diferença $\mathrm{P}(\mathrm{A}-\mathrm{a}) \mathrm{O}_{2}$ obtida com a $\mathrm{PaCO}_{2}$ medida e com a $\mathrm{PaCO}_{2}$ fixada em $40 \mathrm{mmHg}$. Os resultados foram comparados por teste de correlação simples.
Resultados. A pressão parcial de oxigênio e de gás carbônico arterial média foi de $50,8 \pm 10,4$ e $51,7 \pm 6,4 \mathrm{mmHg}$, respectivamente. As médias da $\mathrm{P}(\mathrm{A}-\mathrm{a}) \mathrm{O}_{2}$, da $\mathrm{P}(\mathrm{A}-\mathrm{a}) \mathrm{O}_{2}$ "corrigida" e da mistura venosa foram respectivamente $26,6 \pm 10,2 \mathrm{mmHg}$; $40,4 \pm 10,8 \mathrm{mmHg}$ e $37 \pm 15,2 \%$. 0 coeficiente de correlação entre a mistura venosa e a $P(A-a) 0_{2}$ foi de $0,61 \quad(p<0,0000 I)$ e mistura venosa e $P(A-a) O_{2}$ "corrigida" foi de 0,89 ( $\left.p<0,00001\right)$.

ConclusÃo. A utilização do cálculo da diferença alvéolo-arterial de oxigênio em pacientes cronicamente hipercapneicos tem alcance clínico limitado, porque ele deixa de expressar o diagnóstico de distúrbio das trocas alvéolo-capilares que freqüentemente está associado à hipoventilação alveolar.

Unitermos: Trocas gasosas. Hipercapnia. Mistura venosa. Diferença alvéolo-arterial de oxigênio.

\section{INTRODUÇÃO}

Tradicionalmente utilizamos a gasometria arterial como instrumento de avaliação da habilidade do pulmão para realizar as trocas gasosas. As alterações da $\mathrm{PaO}_{2}$ e da $\mathrm{PaCO}_{2}$ verificadas em paciente no estado de repouso e respirando ar ambiente refletem o grau de participação da ventilação alveolar e das trocas alvéolo-capilares. A relação linear inversa existente entre o comportamento da $\mathrm{PaO}_{2}$ e da $\mathrm{PaCO}_{2}$ nos permite avaliar facilmente se um determinado grau de hipoxemia arterial pode corresponder ao grau de hipoventilação alveolar revelado pelos níveis de retenção de $\mathrm{CO}_{2}$, enquanto informação mais específicas a respeito da função alvéolo-capilar, como identificação da presença de "shunt", pode ser obtida com a análise do comportamento

\footnotetext{
*Correspondência:

Divisão de Pneumologia do Hospital das Clínicas da Faculdade de Medicina de Ribeirão Preto da

Universidade de São Paulo (HCFMRP/USP) Avenida Bandeirantes, s/n - Campus Universitário Monte Alegre - CEP: 14048-900 - Ribeirão Preto São Paulo - Brasil - Fone: (016) 602-2226 E-mail:gadsilva@fmrp.usp.br.
}

da $\mathrm{PaO}_{2}$ após inalação de oxigênio a 100\% por 15-20 minutos!. Todos os mecanismos funcionais pulmonares que alteram os gases arteriais ocorrem na doença pulmonar obstrutiva crônica.

Outro indicador importante da função de trocas gasosas pulmonares que pode ser obtido rapidamente da gasometria arterial é o cálculo da diferença alvéolo-arterial de oxigênio $\mathrm{P}(\mathrm{A}-\mathrm{a}) \mathrm{O}_{2}$. Ele está aumentado somente nos distúrbios alvéolo-capilares²; auxilia no diagnóstico e na quantificação desta funçãa 3 3.,.5, de forma mais sensível que a medida da $\mathrm{PaO}_{2}$ isolada. $\mathrm{O}$ cálculo da diferença de $\mathrm{PaO}_{2}$ entre os alvéolos e o sangue arterial pressupõe 0 conhecimento da $\mathrm{PO}_{2}$ alveolar que é obtido através da fórmula da pressão parcial de oxigênio alveolar: $\mathrm{PAO}_{2}=\mathrm{PIO}_{2}-\mathrm{PACO}_{2} / \mathrm{R} \cdot \mathrm{Na}$ prática a fórmula é universalmente utilizada ${ }^{6}$ com várias suposições: I) a pressão parcial de $\mathrm{CO}_{2}$ alveolar entra em equilibrio com o sangue, e a partir dessa suposição a $\mathrm{PACO}_{2}$ é substituída pela $\mathrm{PCO}_{2}$ arterial e 2) a taxa de trocas respiratórias, "R", é adotado como sendo 0,8 se o paciente respira ar ambiente ou I,0 se ele respira uma mistura com oxigênio adicional ${ }^{1,2}$. Então, em ar ambiente, a $\mathrm{P}(\mathrm{A}-\mathrm{a}) \mathrm{O}_{2}$ $=\mathrm{PIO}_{2}-\left(\mathrm{PaCO}_{2} \times 1,25\right)-\mathrm{PaO}_{2}$. Essa diferença altera-se precocemente nos processos pulmonares causadores de hiperventilação alveolar ${ }^{7}$ com hipoxemia iminente ou mesmo, francamente ausente, como nas doenças insidiosas do insterstício pulmonar do tipo pneumonia intersticial por Pneumocystis carinii, entre outras. Por outro lado, o cálculo, como utilizado na prática, é enganoso quando empregado em indivíduos hipercapneicos. Esta observação vem de encontro à descrita por $\mathrm{Comroe}^{8}$, segundo a qual a diferença alvéolo-capilar de pressão parcial de oxigênio sofre influência profunda da $\mathrm{PO}_{2}$ alveolar, revelando-se maior quando a $\mathrm{PAO}_{2}$ é maior, sofrendo redução quando há diminuição da $\mathrm{PAO}_{2}$ como na hipoventilação alveolar.

O cálculo da mistura venosa, embora também envolvendo várias suposições, pode constituir uma alternativa de avaliação das trocas alvéolo-capilares, mais fiel que o cálculo da diferença alvéolo-arterial de oxigênio9. Este cálculo está baseado na diferença de conteúdo artério-venoso de oxigênio o qual reflete totalmente a intensidade da hipoxia, mesmo 
quando os níveis de oxigênio do paciente (expressos pela $\mathrm{PaO}_{2}$ e saturação da oxihemoglobina) estão na faixa íngreme da curva de saturação. $O$ mesmo não ocorre com a pressão parcial do gás, que é função da quantidade dissolvida no plasma, que por sua vez sofre somente pequenas alterações face a consideráveis quedas na quantidade de oxigênio combinado à hemoglobina.

Propomos que a mistura venosa é um indicador mais fiel da eficiência pulmonar em efetuar trocas gasosas, independente do mecanismo envolvido. No presente trabalho, comparamos o valor numérico da mistura venosa com o da $\mathrm{P}(\mathrm{A}-\mathrm{a}) \mathrm{O}_{2}$ usando, a $\mathrm{PaCO}_{2}$ real e a $\mathrm{PaCO}_{2}$ ideal de $40 \mathrm{mmHg}$, de pacientes normo e hipercapneicos. Pretendemos com isso avaliar a eficácia da $\mathrm{P}(\mathrm{A}-\mathrm{a}) \mathrm{O}_{2}$ em relação à mistura venosa, para evidenciar distúrbio das trocas alvéolo-capilares em pacientes com doença pulmonar obstrutiva crônica onde, reconhecidamente, com freqüência ocorrem desigualdades da relação $\mathrm{V} / \mathrm{Q}$, distúrbio de difusão através da membrana alvéolo-capilar e "shunt" associados à hipoventilação alveolar, através da comparação destes dois indicadores, por correlação simples. Não encontramos na literatura nenhum artigo que aborde o tema com enfoque semelhante ao apresentado aqui.

\section{Métodos}

Foram analisadas gasometrias arteriais de 83 pacientes com pressão parcial de gás maior que $40 \mathrm{mmHg}$, portadores de doença pulmonar obstrutiva crônica, adultos, de ambos os sexos. A gasometria foi obtida por punção da artéria radial utilizando-se seringa heparinizada com solução 1:1000 de heparina, de vidro, o que permitia que a própria pressão sangüínea elevasse 0 êmbolo da seringa, assegurando a origem arterial do sangue coletado. A seringa foi imediatamente ocluída após a coleta e, em seguida, levada para análise ou foi mantida sob temperatura de $2^{\circ}$ a $4^{\circ} \mathrm{C}$ até o momento da análise ${ }^{10}$. Evitou-se a formação de pequenas bolhas na seringa, apesar do seu efeito insignificante sobre a gasometria arterial". Foi feita também uma determinação da quantidade de hemoglobina destes pacientes.

\begin{tabular}{|c|c|c|c|c|c|c|c|}
\hline & $\begin{array}{l}\mathrm{Hb} \\
\mathrm{g} / \mathrm{dl}\end{array}$ & $\begin{array}{c}\text { Sat } \mathbf{O}_{2} \\
\%\end{array}$ & $\mathrm{PaO}_{2}$ & $\begin{array}{l}\mathrm{PaCO}_{2} \\
\mathrm{mmHg}\end{array}$ & $\mathrm{P}(\mathrm{A}-\mathrm{a}) \mathrm{O}_{2}$ & $\begin{array}{c}\text { Qs/Qt } \\
\%\end{array}$ & $\begin{array}{c}\mathrm{P}(\mathrm{A}-\mathrm{a}) \mathrm{O}_{2} \mathrm{C} \\
\mathrm{mmHg}\end{array}$ \\
\hline $\begin{array}{l}\text { Média } \\
\text { Mediana } \\
\text { V. mínimo } \\
\text { V. máximo } \\
\text { DP }\end{array}$ & $\begin{array}{l}14,7 \\
14,4 \\
10,4 \\
19,7 \\
2,2\end{array}$ & $\begin{array}{l}82,2 \\
84,2 \\
26,0 \\
95,0 \\
10,8\end{array}$ & $\begin{array}{l}50,8 \\
50,7 \\
20,6 \\
79,4 \\
10,4\end{array}$ & $\begin{array}{c}51,7 \\
51,0 \\
44,0 \\
78,3 \\
6,4\end{array}$ & $\begin{array}{l}26,6 \\
26,5 \\
-1,3 \\
51,0 \\
10,2\end{array}$ & $\begin{array}{l}37,0 \\
37,9 \\
9,36 \\
75,2 \\
15,2\end{array}$ & $\begin{array}{l}40,4 \\
41,0 \\
12,0 \\
70,6 \\
10,8\end{array}$ \\
\hline
\end{tabular}

Legenda: $\mathrm{Hb}=$ hemoglobina, Sat $\mathrm{O}_{2}=$ saturaçãão da oxihemoglobina, $\mathrm{PaO}=$ = pressão parcial de oxigênio arterial, $\mathrm{PaCO}_{2}=$ pressão parcial de gás carbồnico arterial, $\mathrm{P}(\mathrm{A}-\mathrm{a}) \mathrm{O}_{2}=$ diferenç̧a alvéolo-arterial de oxigênio para $\mathrm{PaCO}$, real, Qs/Qt=mistura venosa; $\mathrm{P}(\mathrm{A}$-a $) \mathrm{O}_{2} \mathrm{c}=$ diferenç̧a alvéolo-arterial de oxigênio com $\mathrm{PaCO}_{2}$ corrigido para o valor ideal de $40 \mathrm{mmHg}, \mathrm{DP}=$ desvio padrão.

O cálculo da $\mathrm{P}(\mathrm{A}-\mathrm{a}) \mathrm{O}_{2}$ e da mistura venosa foram obtidos a partir de um programa de computador desenvolvido na nossa Disciplina, contendo banco de dados e as equações: a) do ar alveolar para respiração em ar ambiente ${ }^{2}$, ou seja, era inserida a pressão barométrica do dia, na nossa cidade, para o cálculo da $\mathrm{PIO}_{2}$ e o valor da taxa de trocas respiratórias "R" foi fixado em 0,8; b) da dissociação do oxigênio no sangue humano ${ }^{12}$ e c) da mistura venosa ', destinada especificamente a este estudo. A equação para obtenção da $\mathrm{PAO}_{2}$ foi:

$\mathrm{PAO}_{2}=\mathrm{F}_{1} \mathrm{O}_{2}(\mathrm{~PB}-47)-\mathrm{P}_{\mathrm{A}} \mathrm{CO}_{2} \times\left[\mathrm{F}_{1} \mathrm{O}_{2}+\right.$ $\left.\left(\mathrm{I}-\mathrm{F}_{1} \mathrm{O}_{2}\right) / 0,8\right]$

Todas as gasometrias do estudo foram colhidas com o paciente respirando ar ambiente, razão pela qual utilizamos a taxa de trocas respiratórias de 0,8 conforme universalmente utilizado na prática. Para 0 cálculo da $\mathrm{P}(\mathrm{A}-\mathrm{a}) \mathrm{O}_{2}$ subtraimos da $\mathrm{PAO}_{2}$ a $\mathrm{P}_{\mathrm{a}} \mathrm{O}_{2}$.

O valor da mistura venosa foi obtido a partir da chamada equação do shunt: Qs/Qt $=\left(\mathrm{Cc}_{\mathrm{O} 2}^{\prime}-\mathrm{Ca}_{\mathrm{O} 2}\right) /\left(\mathrm{Cc}_{\mathrm{O} 2}^{\prime}-\mathrm{Ca}_{\mathrm{O} 2}\right)+$ $\left(\mathrm{Ca}_{\mathrm{O} 2}-\mathrm{Cv}_{\mathrm{O} 2}\right)$, onde Qs é a quantidade de sangue que alcança a circulação sistêmica sem sofrer hematose por minuto e Qt é a quantidade total de sangue que passa pelo pulmão por minuto. $\mathrm{CC}_{02}^{\prime}$ é o conteúdo de oxigênio capilar, $\mathrm{Ca}_{\mathrm{O} 2}$ é o conteúdo de oxigênio arterial e $\mathrm{CV}_{\mathrm{O}_{2}}$ é o conteúdo de oxigênio venoso misto. A diferença de conteúdo artério-venoso de oxigênio foi considerada fixa e igual a $4,5 \mathrm{ml} / \mathrm{dl}$, uma vez que asseguramos a não-inclusão de pacientes com cardiopatias congênitas ou outra forma de cardiopatia descompensada.
O conteúdo capilar de oxigênio foi obtido a partir da saturação da hemoglobina para $\mathrm{PAO}_{2}$ calculada, para $\mathrm{PaCO}_{2}$ e pH observados, e temperatura corporal de $37^{\circ} \mathrm{C}$ e levando em conta o valor observado da hemoglobina ${ }^{12}$.

Os valores obtidos da $\mathrm{P}(\mathrm{A}-\mathrm{a}) \mathrm{O}_{2}$ com a $\mathrm{PaCO}_{2}$ real e com a $\mathrm{PaCO}_{2}$ corrigida foram comparados com os valores da mistura venosa por correlação simples.

\section{Resultados}

Todos os pacientes eram adultos, 43 do sexo masculino, todos com diagnóstico clínico e funcional de doença pulmonar obstrutiva crônica grave ou muito grave de acordo com o Consenso Brasileiro da Sociedade Brasileira Pneumologia ${ }^{13}$.

A pressão parcial de oxigênio e de gás carbônico arterial média foi de 50,8 $\pm 10,4$ e $51,7 \pm 6,4 \mathrm{mmHg}$, respectivamente. As médias da $\mathrm{P}(\mathrm{A}-\mathrm{a}) \mathrm{O}_{2}$, da $\mathrm{P}(\mathrm{A}-\mathrm{a}) \mathrm{O}_{2}$ "corrigida" e da mistura venosa foram respectivamente $26,6 \pm 10,2 \mathrm{mmHg} ; 40,4 \pm 10,8$ $\mathrm{mmHg}$ e $37 \pm 15,2 \%$. O coeficiente de correlação entre a Qs/Qt e a $\mathrm{P}(\mathrm{A}-\mathrm{a}) \mathrm{O}$, fol de 0,6I $\left(r^{2}=0,37\right) ; p<0,00001$ e Qs/Qt e $\mathrm{P}(\mathrm{A}-\mathrm{a}) \mathrm{O}_{2}$ "corrigida" foi de $0,89\left(\mathrm{r}^{2}=\right.$ $0,80) ; p<0,00001$. Esses valores, além da mediana, dos valores mínimo e máximo e desvios-padrão encontrados para essas variáveis encontram-se na Tabela I. O Gráfico I ilustra a distribuição e o coeficiente de correlação entre os valores da mistura venosa e da $\mathrm{P}(\mathrm{A}-\mathrm{a}) \mathrm{O}_{2}$ real. O Gráfico 2 ilustra a distribuição e o coeficiente de correlação entre a mistura venosa e a $\mathrm{P}(\mathrm{A}-\mathrm{a}) \mathrm{O}$, "corrigida" para $\mathrm{PaCO}_{2}$ ideal de $40 \mathrm{mmHg}$. 
Gráfico I - Distribuição da correlação entre os valores da mistura venosa e da diferença alvéolo-arterial de oxigênio calculada com o valor real da pressão parcial de gás carbônico, em pacientes hipercapneicos

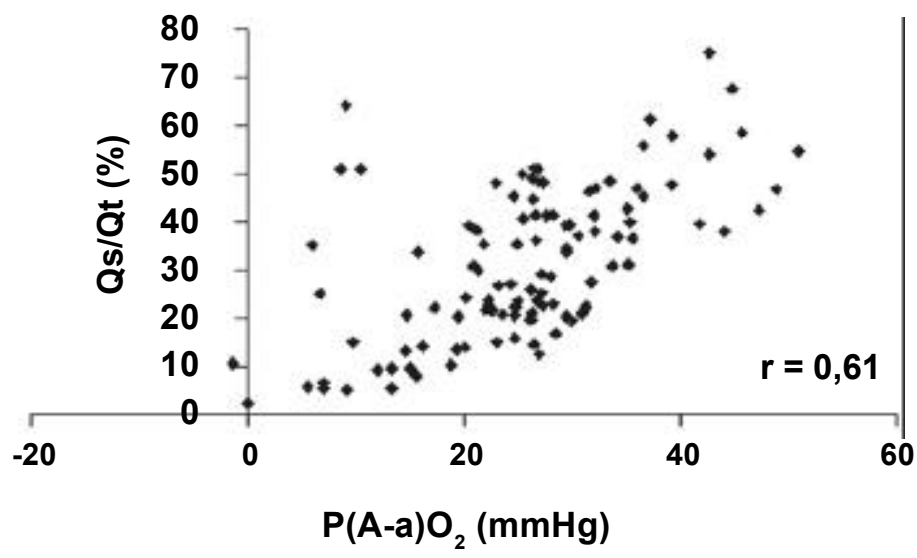

Legenda: $\mathrm{P}(\mathrm{A}-\mathrm{a}) \mathrm{O}_{2}=$ diferença alvéolo-arterial de oxigênio, $\mathrm{Qs} / \mathrm{Qt}=$ mistura venosa.

Gráfico 2 - Distribuição da correlação entre os valores da mistura venosa e da diferença alvéolo-arterial de oxigênio calculada com ovalor "corrigido" da pressão parcial de gás carbônico, em pacientes hipercapneicos

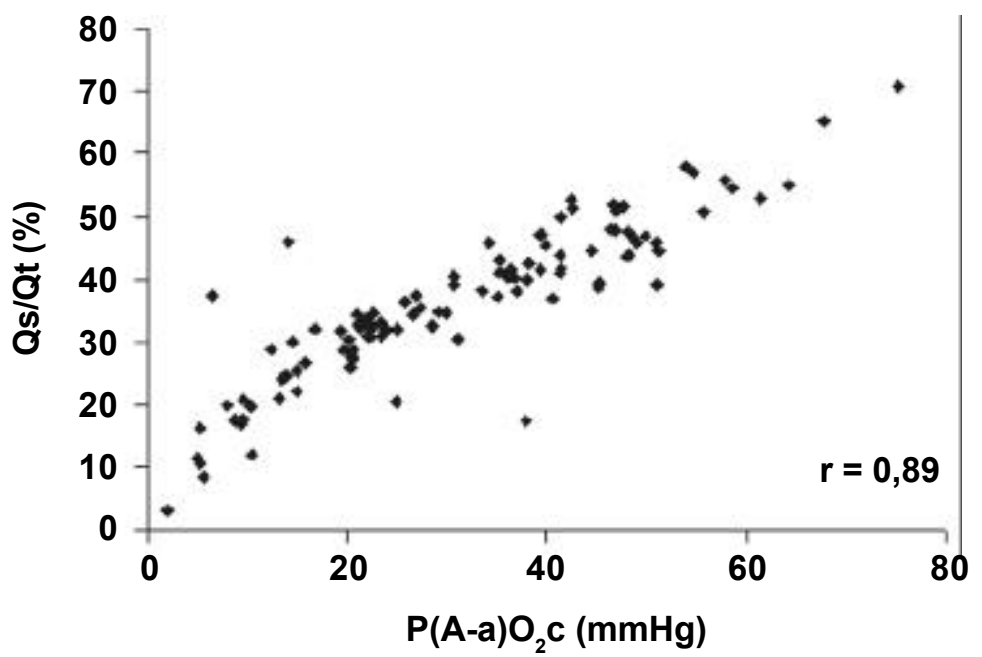

Legenda: $\mathrm{P}(\mathrm{A}-\mathrm{a}) \mathrm{O}_{2} \mathrm{c}=$ diferença alvéolo-arterial de oxigênio "corrigida" para $\mathrm{PaCO}_{2}$ de $40 \mathrm{mmHg}$. Qs/Qt=mistura venosa

\section{Discussão}

A diferença alvéolo-arterial de oxigênio normal decorre de dois mecanismos principais $^{2}$ que são: a) as desigualdades da relação ventilação-perfusão, que são próprias do pulmão mesmo nas condições de normalidade funcional e b) a mistura venosa. Esta representa uma parte do sangue venoso misto que alcança a circulação arterial sistêmica (veias pulmonares, átrio esquerdo e ventrículo esquerdo) através da circulação brônquica e veias de Thebésiun, sem passar pelas trocas gasosas pulmonares.

A quantidade da mistura venosa é anormalmente alta em certas doenças congênitas do coração e quando há comunicações artério-venosas pulmonares, ${ }^{1,2}$ como na doença de Rendu-Osler-Weber. Sob tais circunstâncias a diferença alvéolo-arterial de oxigênio também é alta e a $\mathrm{PaO}_{2}$ é reduzida enquanto a $\mathrm{PaCO}_{2}$ é freqüentemente mais baixa que 0 normal, porque a hipoxemia induz à hiperventilaçao. Adicionalmente, o aumento de áreas pulmonares com baixa relação ventilção/ perfusão, como áreas de atelectasia, edema pulmonar ou consolidação pulmonar, simularão um aumento da mistura venosa.

O cálculo da diferença alvéolo-arterial de oxigênio envolve o cálculo da pressão parcial do oxigênio alveolar, através da seguinte fórmula: $\mathrm{PAO}_{2}=\mathrm{PIO}_{2}-\mathrm{PACO}_{2} / \mathrm{R}$. Esse por sua vez envolve a suposiçãa de que a pressão parcial de gás carbônico alveolar equilibra-se com a capilar pulmonar e que este se mantém igual até atingir a artéria periférica onde 0 sangue é coletado, e a suposição de que a taxa de trocas respiratórias é igual a 0,8 se o paciente respira ar ambiente, ou igual a um se ele respira uma mistura com uma concentração maior de oxigênio². Então, na prática, a $\mathrm{PACO}_{2}$ é substituída pela $\mathrm{PaCO}_{2}$, que será multiplicada por I,25 (ou PaCO $/ 2,8$ ) sob respiração de ar ambiente. A pressão parcial do oxigênio no ar inspirado $\left(\mathrm{PIO}_{2}\right)$ é função da fração de oxigênio no ar inspirado e da pressão barométrica; é também calculada através da seguinte fórmula: $\mathrm{PIO}_{2}=\mathrm{FIO}_{2}$ (PB-47) onde 47, expresso em $\mathrm{mmHg}$, é a saturação do vapor d'agua na temperatura corporal de $37^{\circ} \mathrm{C}$. O valor normal da $\mathrm{P}(\mathrm{A}-\mathrm{a}) \mathrm{O}_{2}$ varia com a idade ${ }^{6}$, essencialmente por causa da queda da $\mathrm{PaO}_{2}$ com a idade, em decorrência da perda de complacência pulmonar que leva à deterioração da distribuição da ventilação.

Da mesma forma, o cálculo da mistura venosa leva em conta diversas suposições, o que o torna, na prática, semelhante ao cálculo da diferença alvéolo-arterial de oxigênio, utilizando entretanto o conteúdo de oxigênio em vez da pressão parcial do oxigênio. Na fórmula da mistura venosa, o conteúdo de oxigênio capilar é calculado a partir da $\mathrm{PO}_{2}$ alveolar obtida pela equação do ar alveolar $1,2,8$. A pressão parcial do oxigênio no sangue venoso misto $\left(\mathrm{Pv}^{-} \mathrm{O}_{2}\right)$ é usualmente determinada de forma direta a partir de amostra de sangue venoso misto coletado no ventrículo direito ou no tronco da artéria pulmonar, por cateterismo cardíaco, quando a finalidade é diagnosticar e medir "shunt" extrapulmonar. Na ausência de cardiopatias congênitas e de insuficiência circulatória, a diferença de conteúdo artério-venoso de oxigênio costuma ser estimada $1.9 \mathrm{em}$ $4,5 \mathrm{ml} / \mathrm{dl}$. O único elemento realmente medido passa a ser a pressão parcial do oxigênio. 
O uso freqüente e continuado dos recursos laboratoriais disponíveis para investigação das doenças respiratórias nos remeteu a diversas leituras sobre o real significado da diferença alvéolo-arterial de oxigênio na prática clínica. Todos que se utilizam dessa medida na rotina com freqüência se deparam com dois tipos de questão, que são: a) como essa diferença pode apresentar valores negativos ocasionalmente e b) como alguns pacientes com DPOC, com gasometria arterial visivelmente alterada, mantêm uma diferença alvéolo-arterial de oxigênio normal? Certamente a resposta para a primeira pergunta reside em "armadilhas" do método; uma diferença negativa de oxigênio entre os alvéolos e o sangue capilar só poderia existir por um curto intervalo de tempo, correspondente à adaptação do pulmão a alguma condição específica. Quanto à segunda pergunta, nos ocorreu tentar respondê-la através do alerta para o fato de que a equação precisa ser interpretada com cautela quando aplicada a pacientes com hipoventilação alveolar, pelo menos da maneira prática como todos a utilizam, qual seja, sem a correção da taxa de trocas respiratórias "R", para desigualdades na distribuição da ventilação. Raras circunstâncias usam coletar gás expirado enquanto se coleta gasometria arterial. Corroborando com essa hipótese, há uma descrição de $\mathrm{Cotes}^{8}$, do diagrama $\mathrm{O}_{2}-\mathrm{CO}_{2}$ para pacientes com insuficiência respiratória, mostrando a relação linear entre os valores médios para as pressões parciais dos gases alveolares e do sangue arterial e efeito da mistura venosa, obtidos por dois autores, em 487 pacientes com doença pulmonar. A pressão parcial de oxigênio alveolar $\left(\mathrm{PAO}_{2}\right)$ apresentada foi calculada pela equação do ar alveolar com a taxa de trocas respiratórias "R" estimada em 0,83 . Seus dados mostram que pacientes com altas pressões parciais de dióxido de carbono arterial podem ter uma menor diferença alvéolo-arterial de oxigênio, mas uma maior mistura venosa que os pacientes com baixas pressões parciais de dióxido de carbono, em que a ventilação alveolar, e portanto a pressão parcial de oxigênio alveolar é mais alta, concluindo que os dois índices nem sempre são intercambiáveis.

A hipoventilação alveolar é uma ventilação inadequada em relação à produção de $\mathrm{CO}_{2}$, quebrando o equilíbrio que se expressa na relação que existe entre ventilação alveolar (V̊A), produção de gás carbônico $\left(\mathrm{VCO}_{2}\right)$ e a pressão parcial do gás carbônico $\left(\mathrm{PaCO}_{2}\right)$, através da seguinte equação: $\vee \mathrm{V}=0,863 \cdot \mathrm{VCO}_{2} / \mathrm{PaCO}_{2}$, onde 0,863 é o fator que corrige para o fato de que a produção de gás carbônico é expressa como um volume de gás seco sob temperatura e pressão "Standard" (STPD), enquanto a ventilação alveolar é expressa como um volume de gás úmido sob temperatura e pressão corporais (BTPS)2.

As alterações de relação ventilação/ perfusão que, em última instância, englobam todos os mecanismos capazes de alterar as trocas gasosas pulmonares são capazes de alterar a composição do gás expirado levando a uma falsa expressão da taxa de trocas gasosas. Tal percepção originou no passado a proposição da determinação do índice de trocas respiratórias por Read ${ }^{8}$ para avaliar o efeito da desigualdade da relação ventilação/perfusão sobre a estimativa das trocas gasosas respiratórias, segundo o qual se determina a mudança proporcional na taxa de trocas respiratórias entre amostras de gás expirado, coletado após dois diferentes volumes de ar terem sido expirados, seguindo-se a uma inalação de ar ambiente. As diferentes constantes de tempo pulmonar, descritas por diversos autores, decorrentes de alvéolos que esvaziam mais tarde na expiração terem uma mais baixa relação ventilação/perfusão que aqueles que esvaziam mais cedo, responderiam pela maior parte da aparente mudança no "R" em indivíduos com ventilação desigual.

A DPOC, doença que tem como causa principal o tabagismo prolongado, envolve pessoas de ambos os sexos, na faixa etária a partir dos 40 anos, provocando sofrimento respiratório progressivo até levá-las à morte. O traço fisiopatológico característico é a limitação crônica e irreversível ao fluxo aéreo que pode ser documentado através da medida espirométrica dos indicadores de fluxo aéreo ${ }^{13}$. O estadiamento e avaliação da progressão da doença envolve também a análise da gasometria arterial onde são verificados rotineiramente a pressão parcial de oxigênio e de gás carbônico, o pH, os níveis de bicarbonato, o excesso de base e a saturação da oxihemoglobina. Todos os mecanismos pulmonares geradores de hipóxia estão presentes na DPOC; são eles: desigualdades da distribuição da ventilação, defeitos da difusão, "shunt" e hipoventilação alveolar. Todos esses mecanismos levam à hipoxemia arterial acompanhada, com freqüência, da hipercapnia decorrente de hipoventilação alveolar. A diferença alvéolo-arterial de oxigênio costuma estar aumentada nos três primeiros mecanismos ${ }^{1,2}$.

$\mathrm{Na}$ gasometria arterial a pressão parcial do oxigênio e do gás carbônico e o pH são sempre medidos por meio de eletrodos, que constituem o meio mais preciso de análise ${ }^{10}$; os outros três elementos são calculados a partir daqueles elementos medidos.

O valor diagnóstico da gasometria arterial amplia-se no cálculo da diferença alvéolo-arterial de oxigênio e da mistura venosa, pois os dois são clássicos indicadores de disfunção alvéolo-capilar ("shunt", desigualdades da distribuição da ventilação, e defeitos de difusão), na geração de hipoxemia.

O nosso trabalho teve o objetivo de ressaltar a diferença entre o valor dos dois indicadores na presença de hipercapnia. Mostramos que os dois índices guardam boa correlação entre si e que esta correlação melhora sensivelmente quando "idealizamos" uma adequada ventilação alveolar através da fixação da PACO em 40 mmHg para o cálculo da $\mathrm{P}(\mathrm{A}-\mathrm{a}) \mathrm{O}_{2}$. O raciocínio envolvido no uso da $\mathrm{PACO}_{2}$ corrigida envolve o conceito de que uma baixa ventilação alveolar resulta em má distribuição de ar para locais bem perfundidos no pulmão, gerando piora da relação ventilação/perfusão o que tende a acentuar a dificuldade para trocar oxigênio, em proporções semelhantes.

A escolha dos pacientes com DPOC teve dois motivos principais: a) eles representam a maioria dos pacientes nos quais detectamos a ineficiência da diferença alvéolo-arterial de oxigênio em diagnosticar alterações nas trocas gasosas alvéolo-capilares nas condições de repouso e respiração de ar ambiente; b) eles agregam todos os distúrbios de trocas gasosas pulmonares, principalmente as desigualdades na distribuiç̧ão da ventilaçãao e a hipoventilação alveolar. Todos os exames foram coletados no laboratório, portanto, em pacientes em condições ambulatoriais, com quadro clínico controlado, o que assegura a não-ocorrência de insuficiência cardiocirculatória descompensada, quadros mais comumente encontrados no ambiente das enfermarias ou no centro de terapia intensiva. 


\section{Conclusão}

O uso da diferença alvéolo-arterial de oxigênio em pacientes cronicamente hipercapneicos tem alcance clínico limitado. Ela não evidencia o diagnóstico de distúrbio das trocas alvéolo-capilares (desigualdades na distribuição da ventilação, dificuldades de difusão e "shunt") que com freqüência encontra-se associado aos processos de hipoventilação alveolar e que é responsável por grande parte da hipoxemia apresentada por esses pacientes.

\section{SUMMARY}

\section{Pulmonary gas exchange under ROOM AIR RESPIRATION IN HYPERCAPNEIC PATIENTS}

INTRODUCTION. The alveolar-capillary oxygen difference, $P(A-a) O_{2}$, is important in the study of gas exchange disorders, however does not give us reliable results when applied to hypercapneic patients. On the other hand the venous admixture calculation, seems to be able to diagnose alveolar-capillary gas exchange difficulty of any kind.

OBJECTIVE. Checking the reliability of the alveolar-capillary oxygen difference to diagnose the alveolar-capillary disorder in face of alveolar hypoventilation, by using the venous admixture calculation as standard.

METHODS. 83 blood gas analysis from patients with hypercapnia were submitted to venous admixture and $P(A-a) O_{2}$ determination with the measured $\mathrm{PaCO}_{2}$ and a fixed
$\mathrm{PaCO}_{2}$ of $40 \mathrm{mmHg}$. The results where compared by correlation test.

RESULTS. The mean partial pressure of arterial oxygen and carbon dioxide were respectively, $50.8 \pm 10,4$ and $51.7 \pm 6,4 \mathrm{mmHg}$; the mean value of $P(A-a) O_{2}$, amended $P(A-a) O_{2}$ and venous admixture were respectively $26.6 \pm 10.2$ $\mathrm{mmHg} ; 40.4 \pm 10.8 \mathrm{mmHg}$ and $37 \pm 15.2 \%$. The correlation coefficient between the venous admixture versus $P(A-a) O$, was 0.61 $p<0.0000$ I and the venous admixture versus "amended" $P(A-a) O_{2}$ was $0.89 p<0.00001$.

CONCLUSION. The use of the oxygen alveolar-capillary gradient in chronically hypercapneic patient does not reach the diagnosis of alveolar-capillary gas exchange disorder which usually comes together with alveolar hypoventilation. [Rev Assoc Med Bras 2004; 50(I): 32-6]

KEY WORDS: Gas exchange. Hypercapnia. Venous admixture.Oxygen alveolar-capillary gradient.

\section{REFERÊNCIAS}

I. Foerster II RE, DuBois AB, Briscoe WA, Fisher $A B$. The Lung- physiologic basis of pulmonary function tests. 3rd ed. Chicago: Year Book Medical Publishers, Inc.; 1986. p. I63-89.

2. Cherniack RM, Cherniack L. Respiration in health ad disease. 2 nd ed. Rio de Janeiro: WB Saunders Company, 1983. p.48-75.

3. Manier G, Castaing Y, Guenard H. Determinants of hypoxemia during the acute phase of pulmonary embolism in humans. Am Rev Respir Dis 1985; 132:332-8.
4. Bernard GR, Bradley RB. Adult respiratory distress syndrome: diagnosis and management. Heart Lung 1986; I 5:250-5.

5. Stover DE, Greeno RA, Gagliardi AJ. The use of a simple exercise test for the diagnosis of pneumococystis carinii in patients with AIDS. Am Rev Respir Dis 1989; 139:1343-6.

6. Harris EA, Kenyon AM, Nisbet HD, Seelye ER, Whittock RM. The normal alveolar-arterial oxygen tension gradient in man. Clin Sci Mol Med 1974; 46:89-104

7. Fahri LE, Rahn H. A theoretical analysis of the alveolar-arterial $\mathrm{O}_{2}$ difference with special reference to the distribution effect. J Appl Physiol 1955; 7:699-703.

8. Cotes JE. Lung function. 2nd ed. Oxford: Blackwell Scientific Publications; 1968. p. 161 206

9. Gray BA, Blalock JM. Interpretation of the alveolar-arterial oxygen difference in patients with hypercapnia. Am Rev Respir Dis 1991; 143:4-8.

10. Adams AP, Morgan-Hughes JO, Sykes MK. PH and blood gas analysis: method of measurement and sources of error using electrode systems. Anaesthesia 1967; 22:575-88.

II. Gosling P, Dickson G. Syringe injection pressure: a neglected factor in blood $\mathrm{PO}_{2}$ determination. Ann Clin Biochem 1 990; 27: I 47-5 I.

12. Severinghaus JW. Simple, accurate equations for human blood $\mathrm{O} 2$ dissociation computations. Bethesda: The American Physiological Society; 1979. p.599-602.

13. I Consenso Brasileiro de Doença Pulmonar Obstrutiva Crônica (DPOC). J Pneumol 2000; 26(supl 1):9-10.

Artigo recebido: 12/04/02 Aceito para publicação: 04/I I/03 